\title{
Reduced APRIL Expression Induces Cellular Senescence via a HSPG-Dependent Pathway
}

\author{
Weifeng Ding • Shaoqing Ju • Shengyang Jiang • \\ Li Zhu • Yueguo Wang • Huimin Wang
}

Received: 16 July 2008 / Accepted: 23 April 2009

(C) Arányi Lajos Foundation 2009

\begin{abstract}
APRIL, a member of the TNF superfamily, can induce cell proliferation and is overexpressed in most tumor tissues or cells. Nevertheless, it is still unknown about the effect of decreased levels of APRIL expression on tumor cells. In this study, we analyzed APRIL and HSPG expression in the colon carcinoma cell line, SW480 by Western blot and RT-PCR. And the up-regulation of APRIL and HSPG expression was found in SW480. We also observed that knockdown of APRIL levels in SW480, prominently reversed cell proliferation and partially resulted in senescence phenotypes. Furthermore, cellular senescence due to a decreased level of APRIL expression was associated with engagement of HSPG. Thus, our results suggest that low levels of APRIL play an essential role in cellular senescence via a HSPG-dependent signaling pathway in SW480.
\end{abstract}

Keywords A proliferation-inducing ligand . Cellular senescence - Short hairpin RNA .

Heparan sulfate proteoglycans

W. Ding $\cdot \mathrm{S}$. Ju $\cdot$ Y. Wang $\cdot$ H. Wang $(\bowtie)$

Medical Laboratory Center,

Affiliated Hospital of Nantong University,

No.20, Road Xisi,

Nantong, China

e-mail: ntfyjyk@pub.nt.jsinfo.net

S. Jiang

Public Health School of Nantong University,

Nantong, China

L. Zhu

Institute for Nautical Medicine and Key Laboratory

of Neuroregeneration, Nantong University,

Nantong, China

\author{
Abbreviations \\ APRIL \\ TNF \\ HSPG \\ TACI \\ BCMA \\ BLyS \\ BR3 \\ SA- $\beta$-gal \\ TACI-Fc \\ a proliferation-inducing ligand \\ tumor necrosis factor \\ heparan sulfate proteoglycans \\ transmembrane activator and cyclophilin \\ ligand interactor \\ B-cell maturation antigen \\ B-lymphocyte stimulator \\ BLyS receptor 3 \\ Senescence-associated $\beta$-galactosidase \\ a fusion protein of TACI and the human \\ Fc fragment of immunoglobulin \\ DAPI 2- (4-amidinophenyl )-6-indolecarbamidi- \\ nedihydrochloride \\ PI propidium iodide \\ FCM flow cytometry \\ SDS-PAGE sodium dodecyl sulfate-polyacrylamide \\ gel electrophoresis \\ ECL enhanced chemiluminescence \\ PBS Phosphate-Buffered Saline \\ CRD cysteine-rich domains \\ TNFR TNF receptor \\ RNAi RNA interfering \\ SDC1 syndecan-1 \\ MAPK mitogen-activated protein kinase \\ MKK MAPK kinase kinase
}

\section{Introduction}

Tumor necrosis factor (TNF) is the prototypic member of a family of cytokines with important roles in immune regulation, inflammation, and cancer [1]. Extensive research has shown that there are at least 20 distinct members 
of the TNF superfamily and they exhibit 15-25\% amino acid sequence homology with each other [2]. Except LT $\alpha$, which is expressed only as a soluble molecule, TNF family members are expressed as cell surface proteins acting in a paracrine and autocrine manner.

APRIL (a proliferation-inducing ligand, also known as TRDL-1, TALL-2, and TNFSF13) is a member of the TNF family and has been shown to be capable of inducing the proliferation of certain tumor cell lines in vitro and in vivo [3, 12]. Together with a related member of the TNF family, BLyS (B-lymphocyte stimulator) [15-17], APRIL shares two common receptors, TACI (transmembrane activator and cyclophilin ligand interactor) and BCMA (B-cell maturation antigen) [4, 13, 14]. However, BLyS also binds to BR3 (BLyS receptor 3 or BAFF-R), the least-conserved member of the TNF receptor family [16]. Unlike BLyS, HSPG (heparan sulfate proteoglycans), which can be blocked by heparin, is found to serve as a special receptor or a binding partner for APRIL $[17,18]$. BCMA, TACI, and BR3 are all expressed on B cells, while TACI and BR3 are also detected on the surface of some T cells $[10,16]$. In contrast, HSPG may exist in Jurkat $\mathrm{T}$ cells, fibroblasts, and epithelial cell lines that do not express either BCMA or TACI [13].

Both APRIL and BLyS promote B cell proliferation by binding to BCMA and TACI receptors, but the role of APRIL in immune regulation is not well defined [4-9, 21]. Nonetheless, the biological role of APRIL does not seem to be restricted to proliferation induction [3], but to be more complex, as a proapoptotic effect of APRIL has been demonstrate as well [19, 22]. Furthermore, overexpression in a number of tumors tissues or cell lines, such as colon carcinomas and lung cancer, suggests a regulatory role for APRIL in tumor growth $[3,11]$.

Cellular senescence, the actual age of the cell significantly exceeding the chronological age, can be ascribed to two major types: intrinsic and extrinsic senescence [32]. The term intrinsic senescence is used to describe the process of replicative senescence, which can be triggered by certain oncogenes and tumor suppressors to activate many, well defined pathways, which signal through oncogenes such as Ras, Raf, MEK or c-Myc [23, 29]. Activation of p53 and then through the $\mathrm{p} 53 \rightarrow \mathrm{p} 21 \rightarrow \mathrm{Rb}$ arm to induce senescence appears to be a central pathway of ageing [23, 34]. Moreover, $\mathrm{p} 16^{\mathrm{INK} 4 \mathrm{a}}$ is a part of the canonical $\mathrm{p} 16 / \mathrm{Rb}$ pathway which has long been known to play a role in the replicative senescence, often highly expressed in senescent cells in vitro [29]. siRNA-directed depletion of $\mathrm{p} 16^{\mathrm{INK} 4 \mathrm{a}}$ in human fibroblasts leads to escape from telomere-dependent and independent senescence, raising the possibility of a cross talk between these two pathways, which can be facilitated by p38 [29, 31].

However, the function of APRIL in cellular senescence when it is decreasingly expressed remains unidentified. In the present study, we report that knockdown of APRIL levels in the colon carcinomas cell line, SW480, prominently reversed cell proliferation and partially produced senescence phenotypes. Furthermore, cellular senescence induced by a reduced APRIL level was associated with engagement of HSPG. Our results suggest here that low expression of APRIL may play an essential role in cellular senescence through a HSPG-dependent signaling pathway in colon carcinomas.

\section{Materials and Methods}

Recombinant Plasmids, Cell Line and Relative Reagents

pGCsi/H1/Neo/GFP plasmid vector (Genechem, Shanghai, China) was digested to line by the restriction enzymes, BamH $I$ and Hind III (Promega, San Francisco, California, USA). Four pairs of chemically-synthesized shRNA oligonucleotides of APRIL were inserted into this plasmid through T4 DNA polymerase ligation at $16^{\circ} \mathrm{C}$ for $8 \mathrm{~h}$, and designated pGsh637, pGsh1450, pGsh1534, and pGsh1750. The plasmids, pGEM-APRIL and pGEM-18S, were bacterial expression vectors in which the human APRIL or human 18S RNA PCR products were inserted in the poly dT sites of the pGEM-T Easy vector (Promega), respectively.

The colon carcinoma cell line, SW480 was obtained from ATCC, Manassas, USA.

TACI-Fc, a fusion protein of TACI and the human Fc fragment of immunoglobulin, $(8 \mu \mathrm{g} / \mathrm{ml}$; R\&D, Poway, CA, USA) was used as a competitive reagent to block APRIL binding to TACI.

Heparin (8 U/ml; Pharmacy Department, Affiliated Hospital of Nantong University, China) was used as a blockage reagent for HSPG.

\section{Analysis of APRIL mRNA Level by RTFQ-PCR}

Real-time fluorescence quantitative PCR (RTFQ-PCR) analysis for APRIL was performed by using a LightCycler 1.5 Instrument (Roche, Mannheim, Germany). PCR was performed in a LightCycler capillary in a $20 \mu$ l reaction volume that contained $1 \times$ DNA Master SYBR Green I, $0.2 \mathrm{mM}$ dNTPs, $3.5 \mathrm{mM} \mathrm{MgCl}_{2}, 0.3 \mu \mathrm{M}$ primers, and $2 \mu \mathrm{l}$ cDNA. The PCR protocol was as follows: initial denaturation for $3 \mathrm{~min}$ at $94^{\circ} \mathrm{C}, 40$ cycles at $94^{\circ} \mathrm{C}$ for $6 \mathrm{~s}, 60^{\circ} \mathrm{C}$ for $15 \mathrm{~s}$, and $72^{\circ} \mathrm{C}$ for $12 \mathrm{~s}$. Results were analyzed with LightCycler software, version 3.5.3.

\section{Measurement of mRNA Levels of TACI, HSPG and BCMA by PCR}

Total cellular RNAs were isolated according to the manufacturer's protocol through TRIzol reagent (Invitrogen, Jefferson 
City, MO, USA). cDNAs were generated with $4 \mu \mathrm{g}$ total RNA using the Superscript II reverse transcriptase and oligo d(T) (Fermentas, Burlington, Republic of Lithuania). Each $20 \mu \mathrm{l}$ reverse transcription-PCR (RT-PCR) reaction contained $2 \mu \mathrm{l}$ of the first-strand cDNA, $0.3 \mu \mathrm{M}$ of each primer, $0.2 \mathrm{mM}$ each of dNTP, $1.5 \mathrm{mM} \mathrm{MgCl}_{2}, 1 \times$ polymerase buffer, and 1.5 units of Taq polymerase (Promega). The amplification profile was $30 \mathrm{~s}$ at $94^{\circ} \mathrm{C}, 40 \mathrm{~s}$ at $54^{\circ} \mathrm{C}(\mathrm{TACI}), 58^{\circ} \mathrm{C}(\mathrm{HSPG}), 54^{\circ} \mathrm{C}$ (BCMA), and $56^{\circ} \mathrm{C}\left(\beta_{2} \mathrm{M}\right), 1 \mathrm{~min}$ at $72^{\circ} \mathrm{C}$, followed by a final extension of $10 \mathrm{~min}$ at $72^{\circ} \mathrm{C}$. Reaction products were electrophoresed on a $2 \%$ agarose gel and sequenced.

\section{Generation of Stably Transfected Cell Line}

SW480 cell lines were seeded at $1 \times 10^{5} / 12$ well and the next morning were transiently transfected with lipofectamine 2,000 (Invitrogen), according to the manufacturer's instructions, with pGsh637, pGsh1450, pGsh1534, pGsh1750, or negative control plasmids. After 48 h, cells were screened by neomycin G418 (Invitrogen, $600 \mu \mathrm{g}$ / $\mathrm{ml}$ ) and harvested about 14 days later for the next analysis.

\section{Cell Senescence and Apoptosis Analysis}

SA- $\beta$-gal (Senescence-associated $\beta$-galactosidase) activity test kits were purchased from Genmed Scientifics, Inc. (Arlington, MA, USA). Cells were stained according to the manufacturer's protocol. After SA- $\beta$-gal staining, the percentage of blue cells per 200 cells were observed and calculated under an inverted light microscope (Leica, Germany).

Meanwhile, cellular nuclei were stained by DAPI (Beyotime, Nantong, China). And cell numbers and nuclear sizes were analyzed by Ima-Pro Plus software. Furthermore, cell apoptosis and cell cycle profiles were examined by Flow Cytometry (FCM).

\section{Cell Proliferation Potentia Assay}

Three different cell suspensions were prepared after transfection by pGsh637 plus heparin, or pGsh637 or negative control plasmids. At the same time, a non-transfected cell suspension was prepared as a control. The protocol was as follows: $2 \times 10^{4}$ cells/24 well was as the initial cell number, counted once every $24 \mathrm{~h}$ by microscope after trypsinization for a total of eight times. The cell growth curve profile was drawn by using culture time as the $\mathrm{x}$ axis and cell number as the $\mathrm{y}$ axis. Population doubling time (PDT) was calculated as follow: $P D T=t \times \lg 2 /\left(\lg N_{t}-\lg N_{0}\right)$ [35], t was presented as the culture time, $\mathrm{N}_{\mathrm{t}}$ and $\mathrm{N}_{0}$ were the cell number of the initial time or after culturing for $t$ time. The proliferation assay was repeated three times.
Detection of Protein Levels of APRIL, p16 ${ }^{\mathrm{INK} 4 \mathrm{a}}$ by Western Blot

Cells were washed with PBS, lysed in $50 \mu$ of ice-cold protease inhibitor cocktail and RIPA buffer (Beyotime). Lysates were resolved by 5\% and 12\% SDS-PAGE before transfer to a nitrocellulose membrane (AMC, Ann Arbor, MI, USA). Then membranes were incubated for $4 \mathrm{~h}$ at room temperature with anti-hAPRIL antibody (Protein Tech Group, Chicago, USA) or anti-p16 ${ }^{\mathrm{INK} 4 \mathrm{a}}$ (Acris, Himmelreich, Germany). The primary antibodies were visualized with goat anti-rabbit peroxidase-conjugated antibodies (Sigma, St. Louis, MO, USA) using an enhanced chemiluminescence (ECL) detection system. Blots were quantified by densitometry using acquisition into Adobe Photoshop (Apple, Cupertino, CA, USA) and analyzed with the Bandscan Image software.

Interaction of APRIL and HSPG by Immunoprecipitation

Proteins $(\sim 1 \mu \mathrm{g})$ of APRIL and HSPG were immunoprecipitated with either $1 \mu \mathrm{g}$ HSPG syndecan-1 antibody (Protein Tech Group) or anti-hAPRIL, followed by protein $\mathrm{A} / \mathrm{G}-\mathrm{Sepharose}$ beads for $16 \mathrm{~h}$ at $4^{\circ} \mathrm{C}$. Beads were washed with PBS and eluted by boiling in a SDS-PAGE sample buffer. $1 / 20$ of the eluate was analyzed by Western blot with second antibodies labeled by HRP.

\section{Statistical Analysis}

Statistical significance was tested using a one-factor analysis of variance test for pairs or a non-parametric Wilcoxon test.

\section{Results}

A Prominent Knockdown Effect on APRIL Gene Occurred in the Colon Carcinoma Cell Line, SW480, After pGshAPRIL Transfection

In a previous study, Hahne et al. [3] provided evidence that APRIL was overexpressed in colon carcinoma cell lines. Therefore, we examined a knockdown effect of pGshAPRIL in the colon carcinoma cell line, SW480. Specifically, we constructed four distinct oligonucleotides of pGshAPRIL via a plasmid vector and transfected by using cation liposome particles. At first, considering that the transient transfection effect was very low, only about $14 \%$, we utilized G418 to screen in order to increase the transfection effect. As expected, the average transfection effect was up to approximately $85 \%$ and cells formed good cell clones in 
sharp contrast to that of no screening (Fig. 1a). The subsequent detection of protein level of APRIL in SW480 through Western blot revealed that APRIL had an elevated expression level in SW480 compared with the normal intestinal epithelium cell line, HIEC(unpublished data). At the same time, an exogenous pGsh637 shRNA aimed at the APRIL gene resulted in a prevalent depletion of APRIL protein level compared with control shRNA and so did pGsh1750 $(P<0.01)$, whereas the knockdown capability of pGsh1450 was only about $50 \%$ or pGsh1534 showed almost no knockdown effect on APRIL (Fig. 1b). To confirm these results in a more quantitative fashion, we also performed RTFQ-PCR (Fig. 1c). This analysis closely mirrored the previous result, suggesting that APRIL had an overexpressed level in a colon carcinoma cell line and pGsh637 (hereinafter referred to as pGshAPRIL) had a high knockdown effect on APRIL gene in SW480.


Fig. 1 Knockdown effect after pGshAPRIL transfection in SW480. a Generation of stably transfected cell lines was screened by G418. b Inhibition effect of 4 kinds of plasmids were analyzed by Western blot. Gradation of negative control was fixed to 1 . Data (the numbers under the bands) were presented as a relative fold of the minimum density in each panel. $\beta$-actin was the loading control. c RTFQ-PCR by SRBY Green I. APRIL mRNA level=(copies of APRIL/copies of $18 \mathrm{~S}$ rRNA) $\times 10^{3}$ [35], $18 \mathrm{~S}$ rRNA was the control, $* *, P<0.01$. Representative data from 3 independent experiments were shown
Transfection of pGshAPRIL Deregulates the Growing Potency of the Colon Carcinoma Cell Line

When transfected with pGshAPRIL and counted once every $24 \mathrm{~h}$, we observed that the logarithmic growing time of SW480 cells transfected with pGshAPRIL was between 4 and 7.5 days. In contrast, SW480 cells not transfected with pGshAPRIL or transfected with negative control plasmids had a rapid logarithmic growing time between 3 and 6 days (Fig. 2a). PDT of untransfected group $(1.61 \mathrm{~d})$ was abbreviated than that of transfected group $(2.84 \mathrm{~d}, P<$ $0.05)$. Thus, these data suggested that transfection of pGshAPRIL deregulated the growing potency of the colon carcinoma cell line, SW480, to some degree and indirectly demonstrated that APRIL may induce some tumor cells to proliferate.

Transfection of pGshAPRIL Trigger Cell Senescence of the Colon Carcinoma Cell Line, SW480

The colon carcinoma cells growth rate slowed after transfection with pGshAPRIL vectors. We also observed those cells had a different morphology compared to colon carcinoma cells which were untreated. It was their common characteristic morphology that the cells became more enlarged, flattened, and granular than before, and seemed to age after serial passageing for eight times. Therefore, we stained these cells with SA- $\beta$-gal in order to confirm whether they were senescent or not [29]. Stained pictures demonstrated that the percentage of blue cells was on average up to 67 per 200 cells, but that the negative control or untreated SW480 was about 7 per 200 cells $(P<0.01)$. These results indicated cellular $\beta$-galactosidase activity, a symbol of cell ageing, had enhanced compare with that of no pGshAPRIL-transfection (Figs. 2b, c, 5a). Accumulating evidences implicated transfection of pGshAPRIL induced cell senescence on the colon carcinoma cell line, SW480.

Induction of Cell Cycle Arrest by Exogenous Low-APRIL Due to Transfection of pGshAPRIL in the Colon Carcinoma Cell Line, SW480

To examine the possibility that decreased cell proliferation is the result of apoptotic cell death or cellular senescence induced by low levels of APRIL, we treated SW480 cells with stable transfection of pGshAPRIL and measured cell apoptosis by FCM. Simultaneously, we stained SW480 with DAPI to determine the morphologic change in the cell nucleus.

DAPI staining(Fig. 3d-a) verified that pGshAPRILtreated cells had no nuclear pyknosis or lysis, a characteristic of apoptosis or death (Fig. 3d-b) and FACS scan displayed no statistical significance in apoptotic cell death 

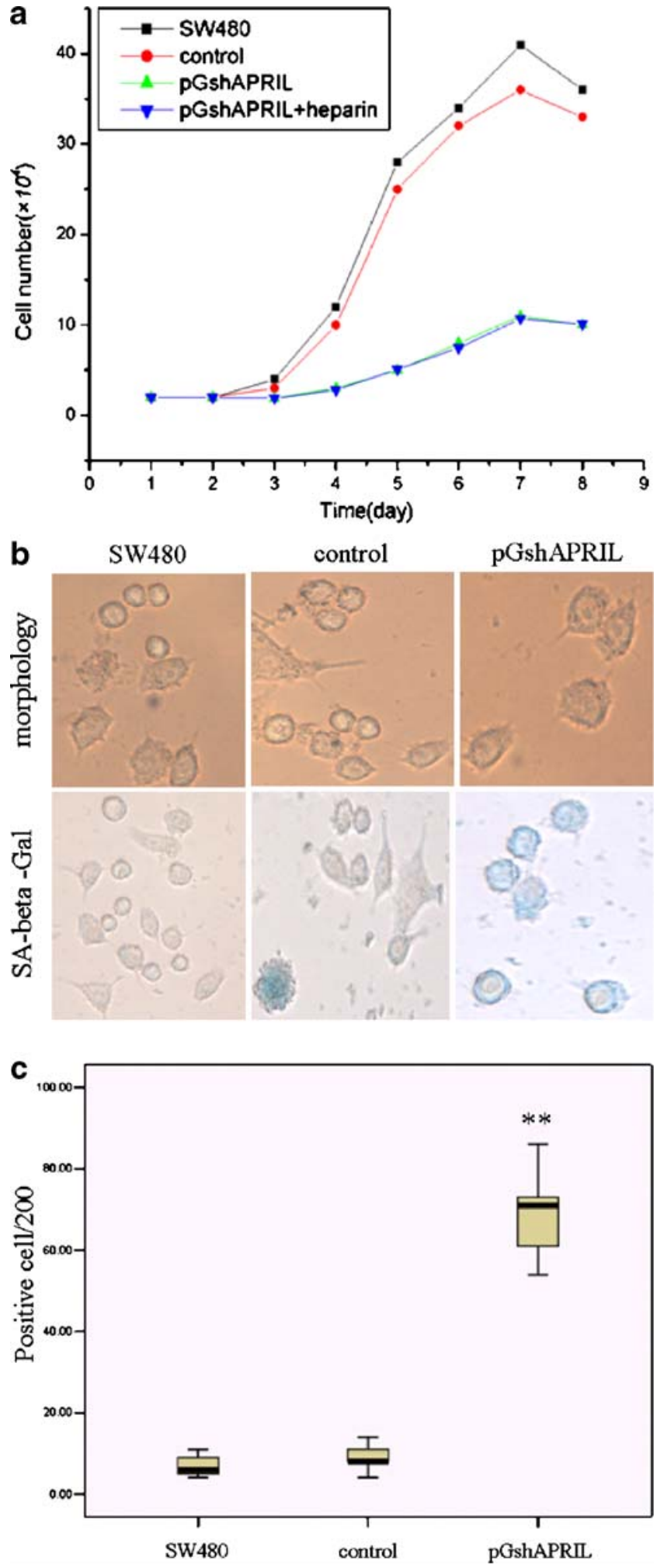

Fig. 2 The growing potency and cellular senescence of SW480 were detected after transfection with pGshAPRIL. a Growth curve profile of SW480 with four kinds of treatment. b Morphology and cellular senescence staining after transfection with pGshAPRIL $(\times 200)$. c Statistics of senile cell number. Values were the means \pm SD of three independent experiments. Representative data from three independent experiments were shown. ${ }^{* *}, P<0.01$ among three groups (Fig. 3a). These evidences demonstrated exogenous low levels of APRIL could not retrieve proliferation into apoptosis. To confirm that pGshAPRILtreated cells entered senescence-related cell cycle arrest, we analyzed DNA content by FCM with PI staining. Most cells that were transfected with pGshAPRIL were arrested in G1 phase (Fig. 3b, c), which is one of the typical phenotypes in cellular senescence [26].

HSPG Serves as a Prominent Receptor of APRIL or Co-Receptor in the Colon Carcinoma Cell Line, SW480

BCMA and TACI are two definitive receptors of APRIL and BLyS [5-8]. However, whether HSPG serves as a third receptor or a binding partner for APRIL is unknown [17, 18]. To discuss the relationship between APRIL and HSPG in SW480 cells, we examined BCMA, TACI, and HSPG mRNA levels by RT-PCR. And then analyzed the potential interreaction between APRIL and HSPG syndecan-1 protein [18] through Co-immunoprecipitation (Co-IP).

RT-PCR revealed that BCMA was not expressed in SW480 at all, while TACI was expressed in trace quantities in SW480. By contrast, SW480 cells had a high level of expression of HSPG (Fig. 4a).Moreover, Co-IP showed APRIL and HSPG had a close interaction with each other in SW480 cell line (Fig. 4b). These findings verified that HSPG serves as a prominent receptor or Co-receptor of APRIL, at least, in colon carcinoma cells.

Induction of Cellular Senescence by Low Levels of APRIL Through a HSPG-Dependent Pathway

To investigate the possibility that HSPG is involved in cell senescence induced by low levels of APRIL, heparin was administered in pGshAPRIL-treated colon carcinoma cells in order to partially block HSPG. Moreover, we used a TACI-Fc fused protein to shield the cellular binding sites, which can interact with APRIL [5, 7], then cells were treated with distinct ways and stained by SA- $\beta$-gal. We also detected the $\mathrm{p} 16^{\mathrm{INK} 4 \mathrm{a}}$ protein level of SW480 cells with different treatment.

It did not show statistical significance in SA- $\beta$-gal activity (low levels) between SW480 groups, heparin and TACI-Fc combined treatment groups, TACI-Fc treatment groups, heparin treatment groups, and control groups (Fig. 5a). By contrast, high levels of SA- $\beta$-gal activity were exhibited in pGshAPRIL-transfected plus TACI-Fctreated groups or those transfected with pGshAPRIL $(P<$ $0.01)$. SA- $\beta$-gal activity was shown to increase in groups with pGshAPRIL and heparin combined treatment and so did in pGshAPRIL, TACI-Fc add heparin treatment groups $(P<0.05)$. Based on these findings, we concluded that pGshAPRIL triggered cell ageing through a HSPGdependent pathway, but not a TACI-dependent mechanism. 
Fig. 3 Cell cycle and apoptosis analysis by flow cytometry and fluorescence microscope. a Cell apoptosis profile was analyzed by FCM with Hoechst 33342 and PI staining. b Cell cycle profile was examined by FCM with PI staining; Cell number were counted according to DNA content of $\mathrm{G} 0 / \mathrm{G} 1, \mathrm{~S}$, and $\mathrm{G} 2 / \mathrm{M}$ phases. c Statistics of cell number of $\mathrm{G} 0 / \mathrm{G} 1, \mathrm{~S}$ and $\mathrm{G} 2 / \mathrm{M}$ phases in cell cycle, ${ }^{*}, P<0.05$. d The cellular nuclear was stained by $\operatorname{DAPI}(a)$ and the cellular diameter was measured. Representative data from three independent experiments were shown. \#, $P>0.05(b)$
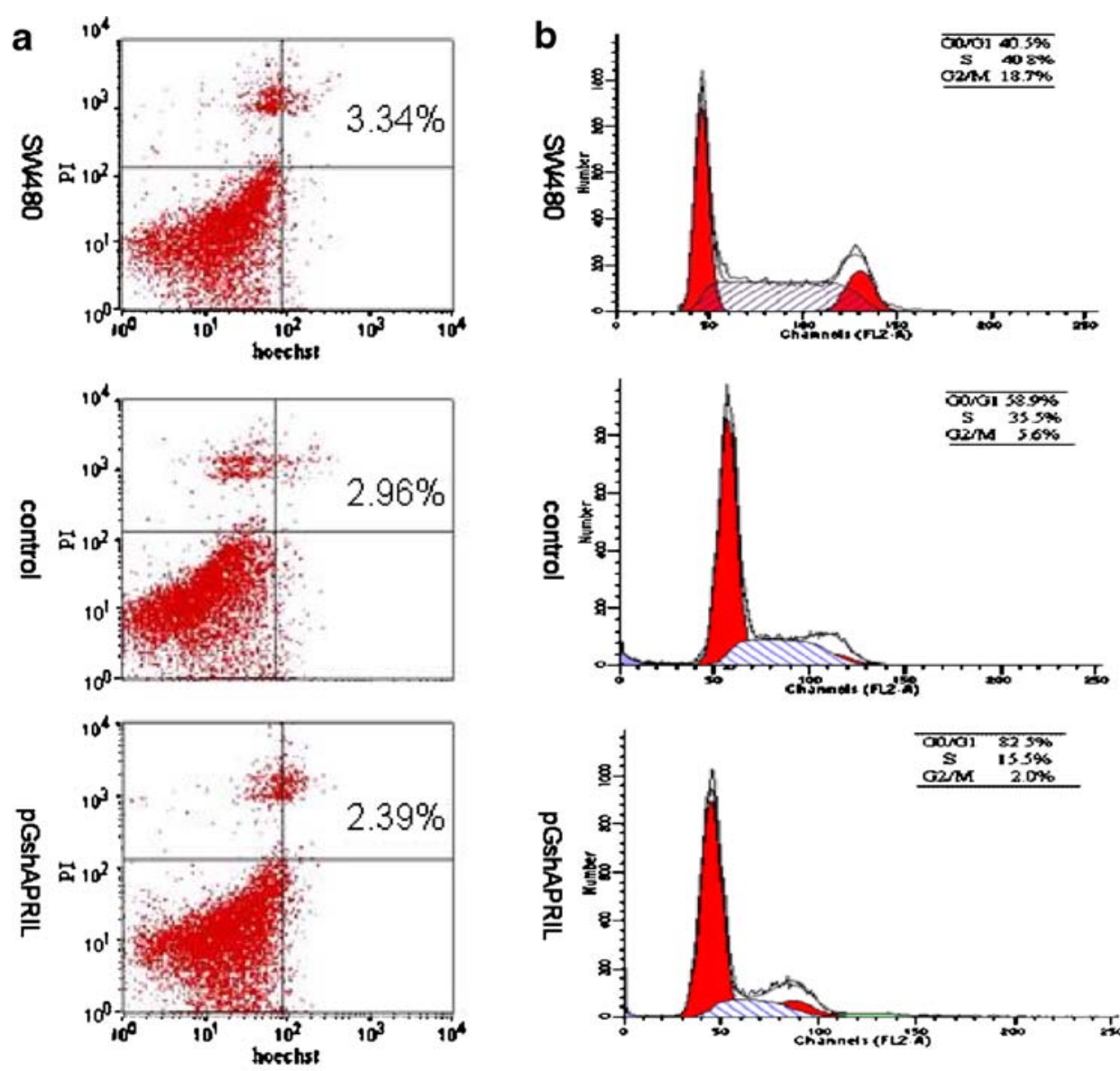

C

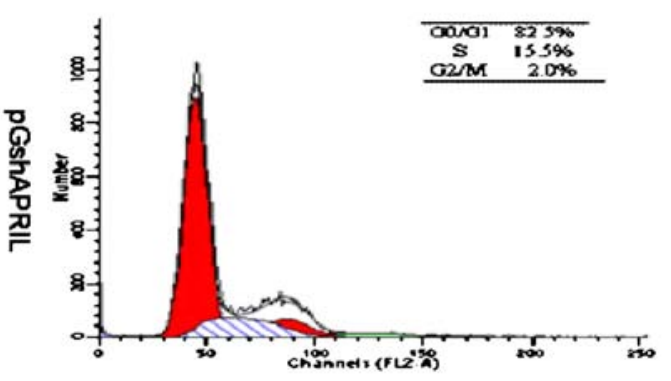

d

DAPI

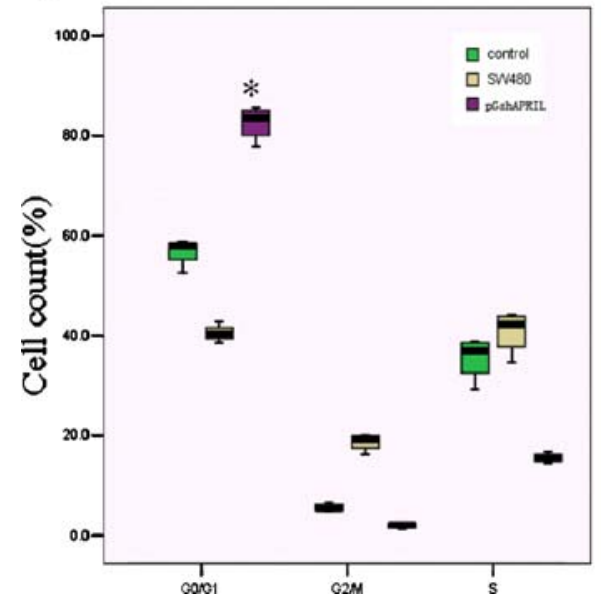

a) SW480 control

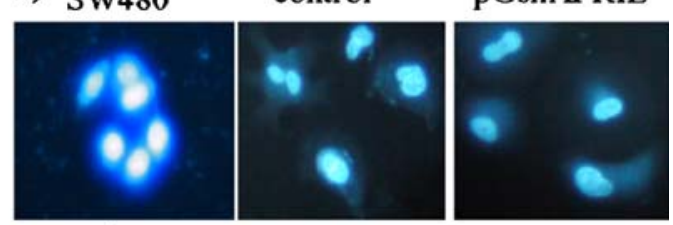

b)

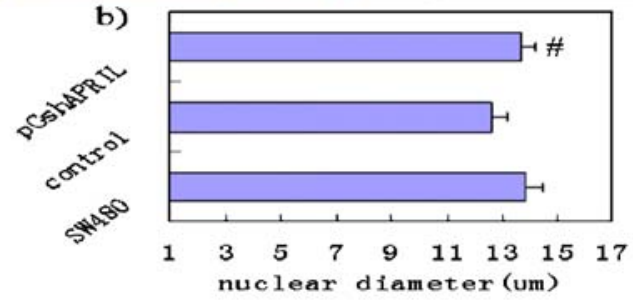

Furthermore, this cellular senescence may be associated with $\mathrm{p} 16 / \mathrm{Rb}$ pathway because that $\mathrm{p} 16^{\mathrm{INK} 4 \mathrm{a}}$ levels were upregulated in response to pGshAPRIL or heparin treatment cells with different degree (Fig. 5b middle panels).

\section{Discussion}

APRIL has an important proliferation-inducing effect, including tumor cells growth $[3,6]$. As a member of the
TNF superfamily, APRIL has the classical TNF fold, which can recognize and bind to cysteine-rich domains (CRD) of the TNF receptor (TNFR) family [14, 27]. Nevertheless, two receptors, TACI and BCMA, bind APRIL, but neither is essential for the tumor-promoting effects, suggesting that a third receptor exists [17, 24]. Furthermore, the crystal structure of APRIL not only consists of the TNF fold, but also contains sulfated glycosaminoglycan side chain reaction sites [20]. Moreover, Hematopoietic cells expressing proteoglycans could accumulate APRIL, triggering auto- 


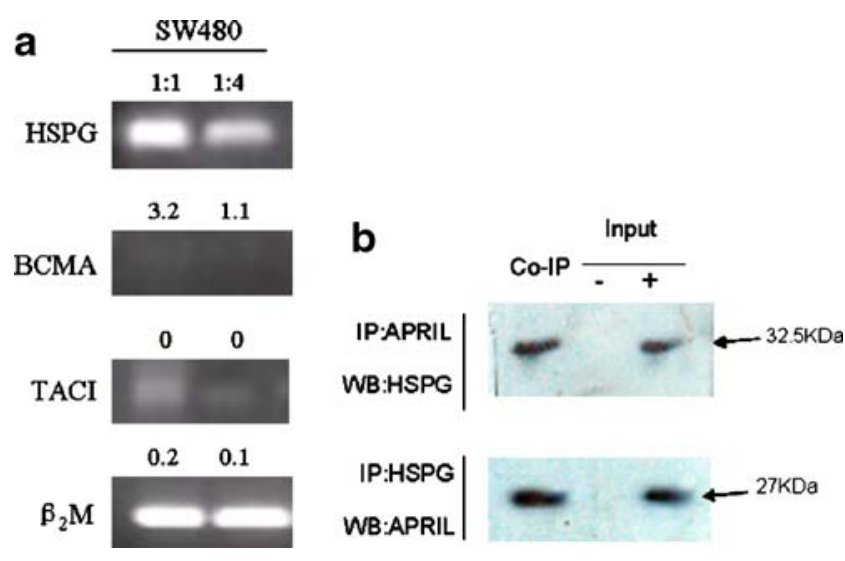

Fig. 4 The Co-relationship between HSPG and APRIL in the colon carcinoma cell line, SW480. a The mRNA level of HSPG, BCMA, and TACI in SW480 and pGshAPRIL transfected cells was detected through RT-PCR. The RNA sample was diluted in $1: 1$ and $1: 4 ; \beta_{2} \mathrm{M}$ served as the control. The density of each band was analyzed and presented as a relative fold of the control density. Representative data from three independent experiments were shown. b The interaction between APRIL and HSPG syndecan-1 protein was examined by coimmunoprecipitation. Input was served as a control

crine growth and tumorigenesis. Transfected Jurkat cells expressing various proteoglycans (syndecan $-1,-2$, or -4 , or the glycolipid-anchored glypican-1) gained robust and specific binding to APRIL. Moreover, two research groups, Ingold $\mathrm{K}$ and Hendriks J, had utilized HSPG syndecan-1 as the representative one of HSPG matrix protein collection to investigate the association between APRIL and HSPG [17, 18], so we did so in this article.

Some researchers have disputed that either HSPG serves as a receptor for APRIL or that HSPG is simply a co-receptor to aid APRIL to interact with its receptors $[17,28]$. In this regard, utilizing HSPG syndecan-1, we demonstrated that the expression of HSPG is increased in SW480 more than TACI and BCMA, which are almost non-existent (Fig. 4a). And on SW480, compact binding capability is existed between native APRIL and HSPG (Fig. 4b). Therefore, we tend to believe that HSPG is a predominant receptor for APRIL, at least, in SW480 cell lines [25]. And we also did not eliminate a possibility that HSPG is only a co-receptor of APRIL due to the limit of the method of Co-IP.

In this study, our experiments demonstrated that the exogenous introduction of pGshAPRIL to the colon carcinoma cell line, SW480, which was overexpressed the APRIL gene, could induce cell senescence to some degree (Figs. 2b, 5a). Simultaneously, not surprisingly, on these SW480 cells with a low level of expression of APRIL, when treated with heparin, blocked the heparin-sulfate sites of APRIL and the cellular senescence phenomenon in SW480 was alleviated (Fig. 5a). Together, these findings showed that a low level of expression of APRIL resulted in cell ageing in SW480 via a HSPG-dependent way.
One critical question pertains to the signal that converts a reversible cell cycle arrest into senescence after knockdown treatment with pGshAPRIL. Alternative interpretations are as follows: First, HSPG expression is regulated by various signaling molecules in vitro, including HGF, EGF, cytokines, and chemotactic factors [30]. We clarified that APRIL was also a cytokine regulator for HSPG (Fig. 4a, b). A previous study has indicated the oncogene Ras was positively expressed in the colon carcinoma cell line, SW480 (ATCC cellline description). Subsequently, we detected p16 levels in several kinds of treated SW480 cells, in view of inactive p53 capacity (mutation in codons 273 and 309) in SW480. A high level of p16 $6^{\text {INK4a }}$ was produced in pGshAPRIL-transfected cells (Fig. 5b). Finally, $\mathrm{p} 14^{\mathrm{ARF}}$ can activate the senescence program by lauching the p53-dependent pathway [29, 32], however, it shows no alteration of $\mathrm{p} 14^{\mathrm{ARF}}$ level in differently treated SW480 cells
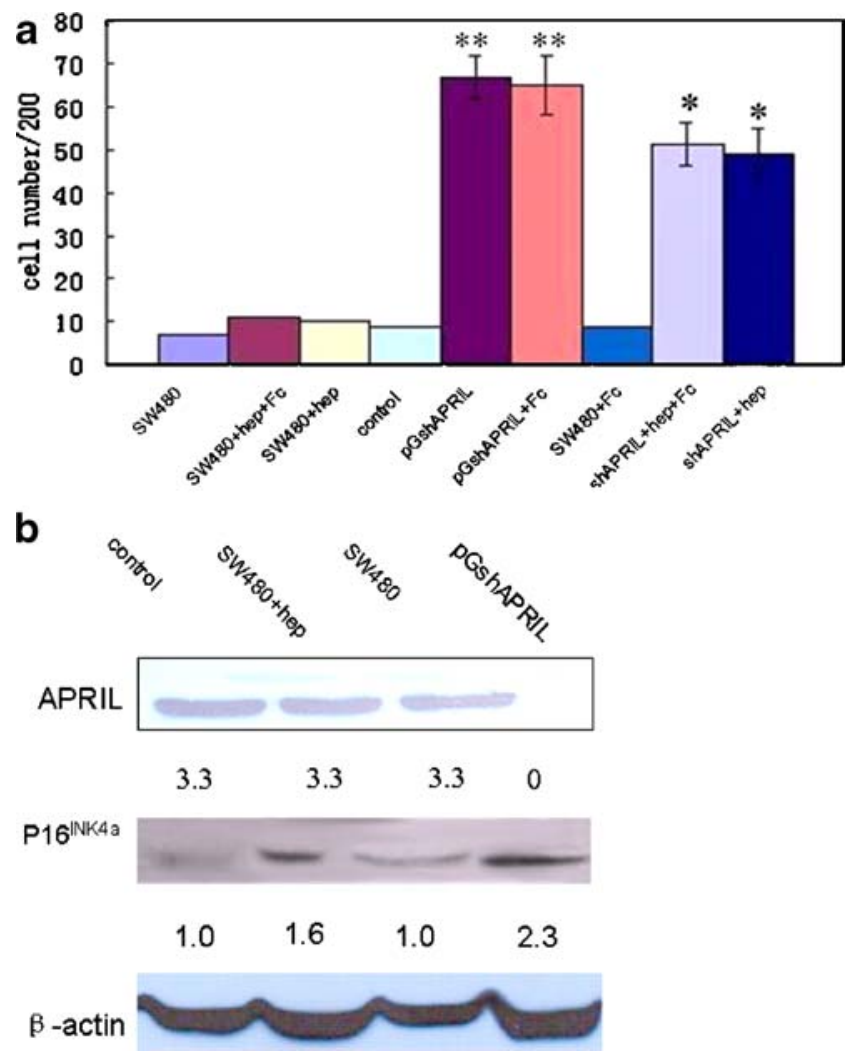

Fig. 5 Cellular senescence was influenced by low level expression of APRIL and HSPG. a Statistics of cell numbers/200 cells. the populations of positive cells were $7 \pm 2,9 \pm 2,10 \pm 2.5$, and $11 \pm 2$ in SW480, SW480+TACI-Fc, SW480+heparin, and SW480+TACI-Fc+ heparin, respectively; the populations of positive cells in pGshAPRIL, pGshAPRIL +TACI-Fc, pGshAPRIL +heparin, and pGshAPRIL + TACI-Fc+heparin were $67 \pm 6,65 \pm 7,49 \pm 5$ and $51 \pm 6$, respectively. Fc, TACI-Fc treatment. hep, heparin treatment. Values were the means \pm SD of three independent experiments. **, $P<0.01 ;{ }^{*}, P<0.05$. b The protein level of APRIL and $116^{\mathrm{INK} 4 \mathrm{a}}$ were analyzed by Western blot. $\beta$ actin was as a control. Representative data from three independent experiments were shown 
(unpublished data). Therefore, $\mathrm{p} 16 / \mathrm{Rb}$ pathway may be an alternative model.

Cellular senescence was associated with an intense activity of SA- $\beta$-gal, a marker for senescent or stressed cultured cells as well as for aged tissues in vivo [32]. Even though $\beta$ galactosidase might be uncharacteristic marker for ageing [33], SW480 cells entered into a growth arrest of G1 phase (Fig. 3b). At the same time, p16 ${ }^{\mathrm{INK} 4 \mathrm{a}}$ and $\mathrm{p} 19^{\mathrm{ARF}}$ (p14 ${ }^{\mathrm{ARF}}$ in the human)-as robust biomarkers of mammalian ageing in almost all tissues [29]-were also detected in this investigation. Therefore, it is conceivable that cellular senescence results from reduced APRIL levels. And it remains us to recreate the signal pathway that induces cellular senescence by exogenous transfection of pGshAPRIL in future experiments.

In conclusion, the results in this study imply that downregulation of APRIL expression by transfection of pGshAPRIL reversed cell proliferation and partially converted a reversible cell cycle arrest into senescence in a HSPG-dependent way, although it was insufficient to induce cell apoptosis. These finding should enhance our understanding of the development of colon carcinomas and provide a new cancer therapy to them.

Acknowledgments The work was supported by the grants from Key Subject of Jiangsu Province (XK20070320). We thank Dr. Xiaoming Zhang for helpful conversations, Dr. David Cushley for assistance with the paper written and are grateful to Prof. Bengt Glimelius and Walter Hoerl for critical reading of the manuscript.

\section{References}

1. Bazzoni F, Beutler B (1996) The tumor necrosis factor ligand and receptor families. N Engl J Med 334:1717-1725

2. Gaur U, Aggarwal BB (2003) Regulation of proliferation, survival and apoptosis by members of the TNF superfamily. Biochem Pharmacol 66:1403-1408

3. Hahne M, Kataoka T, Schroter M, Hofmann K, Irmler M, Bodmer JL, Schneider P et al (1998) APRIL, a new ligand of the tumor necrosis factor family, stimulates tumor cell growth. J Exp Med 188:1185-1190

4. Mackay F, Schneider P, Rennert P, Browning J (2003) BAFF AND APRIL: a tutorial on B cell survival. Annu Rev Immunol 21:231-264

5. Roschke V, Sosnovtseva S, Ward CD, Hong JS, Smith R, Albert $\mathrm{V}$, Stohl W et al (2002) BLyS and APRIL form biologically active heterotrimers that are expressed in patients with systemic immune-based rheumatic diseases. J Immunol 169:4314-4321

6. Nishio M, Endo T, Tsukada N, Ohata J, Kitada S, Reed JC, Zvaifler NJ, Kipps TJ (2005) Nurselike cells express BAFF and APRIL, which can promote survival of chronic lymphocytic leukemia cells via a paracrine pathway distinct from that of SDF1alpha. Blood 106:1012-1020

7. Mongini PK, Inman JK, Han H, Fattah RJ, Abramson SB, Attur $M$ (2006) APRIL and BAFF promote increased viability of replicating human B2 cells via mechanism involving cyclooxygenase 2. J Immunol 176:6736-6751
8. Tangye SG, Bryant VL, Cuss AK, Good KL (2006) BAFF, APRIL and human B cell disorders. Semin Immunol 18:305-317

9. Endo T, Nishio M, Enzler T, Cottam HB, Fukuda T, James DF, Karin M, Kipps TJ (2006) BAFF and APRIL support chronic lymphocytic leukemia B-cell survival through activation of the canonical NF-kappaB pathway. Blood 109:703-710

10. Craxton A, Draves KE, Gruppi A, Clark EA (2005) BAFF regulates B cell survival by downregulating the $\mathrm{BH}$-only family member Bim via the ERK pathway. J Exp Med 202:1363-1374

11. Dillon SR, Gross JA, Ansell SM, Novak AJ (2006) An APRIL to remember: novel TNF ligands as therapeutic targets. Nat Rev Drug Discov 5:235-246

12. Deshayes F, Laprée G, Portier A, Richard Y, Pencalet P, MahieuCaputo D et al (2004) Abnormal production of the TNF-homologue APRIL increases the proliferation of human malignant glioblastoma cell lines via a specific receptor. Oncogene 23:3005-3012

13. Rennert P, Schneider P, Cachero TG, Thompson J, Trabach L, Hertig S, Holler N et al (2000) A soluble form of B cell maturation antigen, a receptor for the tumor necrosis factor family member APRIL, inhibits tumor cell growth. J Expt Med 192:1677-1684

14. Marsters SA, Yan M, Pitti RM, Haas PE, Dixit VM, Ashkenazi A (2000) Interaction of the TNF homologues BLyS and APRIL with the TNF receptor homologues BCMA and TACI. Curr Biol 10:785-788

15. Wu Y, Bressette D, Carrell JA, Kaufman T, Feng P, Taylor K et al (2000) Tumor necrosis factor (TNF) receptor superfamily member TACI is a high affinity receptor for TNF family members APRIL and BLyS. J Biol Chem 275:35478-35485

16. Thompson JS, Bixler SA, Qian F, Vora K, Scott ML, Cachero TG et al (2001) BAFF-R, a newly identified TNF receptor that specifically interacts with BAFF. Science 293:2108-2111

17. Hendriks J, Planelles L, de Jong-Odding J, Hardenberg G, Pals ST, Hahne M, Spaargaren M, Medema JP (2005) Heparan sulfate proteoglycan binding promotes APRIL-induced tumor cell proliferation. Cell Death Differ 12:637-648

18. Ingold K, Zumsteg A, Tardivel A, Huard B, Steiner QG, Cachero TG, Qiang F et al (2005) Identification of proteoglycans as the APRIL-specific binding partners. J Exp Med 201:1375-1383

19. López-Fraga M, Fernández R, Albar JP, Hahne M (2001) Biologically active APRIL is secreted following intracellular processing in the Golgi apparatus by furin convertase. EMBO Rep 2:945-951

20. Wallweber HJ, Compaan DM, Starovasnik MA, Hymowitz SG (2004) The crystal structure of a proliferation-inducing ligand, APRIL. J Mol Biol 343:283-290

21. Varfolomeev E, Kischkel F, Martin F, Seshasayee D, Wang H, Lawrence D, Olsson C et al (2004) APRIL-deficient mice have normal immune system development. Mol Cell Biol 24:997-1006

22. Roth W, Wagenknecht B, Klumpp A, Naumann U, Hahne M, Tschopp J, Weller M (2001) APRIL, a new member of the tumor necrosis factor family, modulates death ligand - induced apoptosis. Cell Death Differ 8:403-410

23. Ben-Porath I, Weinberg RA (2005) The signals and pathways activating cellular senescence. Int J Biochem Cell Biol 37:961-976

24. Chiu A, Xu W, He B, Dillon SR, Gross JA, Sievers E et al (2007) Hodgkin lymphoma cells express TACI and BCMA receptors and generate survival and proliferation signals in response to BAFF and APRIL. Blood 109:729-739

25. Schwaller J, Schneider P, Mhawech-Fauceglia P, McKee T, Myit S, Matthes T et al (2007) Neutrophil-derived APRIL concentrated in tumor lesions by proteoglycans correlates with human B cell lymphoma aggressiveness. Blood 109:331-338

26. Wagner M, Hampel B, Bernhard D, Hala M, Zwerschke W, JansenDürr P (2001) Replicative senescence of human endothelial cells in vitro involves G1 arrest, polyploidization and senescence-associated apoptosis. Exp Gerontol 36:1327-1347 
27. Hymowitz SG, Patel DR, Wallweber HJ, Runyon S, Yan M, Yin J, Shriver SK (2005) Structures of APRIL-receptor complexes: like BCMA, TACI employs only a single cysteine-rich domain for high affinity ligand binding. J Biol Chem 280:7218-7227

28. Schwaller J, Went P, Matthes T, Dirnhofer S, Donze O, MhawechFauceglia P, Myit S, Huard B (2007) Paracrine promotion of tumor development by the TNF ligand APRIL in Hodgkin's Disease. Leukemia 21:1324-1327

29. Famulski KS, Halloran PF (2005) Molecular events in kidney ageing. Curr Opin Nephrol Hypertens 14:243-248

30. Lopes CC, Dietrichand CP, Nader HB (2006) Specific structural features of syndecans and heparan sulfate chains are needed for cell signaling. Braz J Med Biol Res 39:157-167
31. Iwasa H, Han J, Ishikawa F (2003) Mitogen-activated protein kinase p38 defines the common senescence-signalling pathway. Genes Cells 8:131-144

32. Itahana K, Campisi J, Dimri GP (2004) Mechanisms of cellular senescence in human and mouse cells. Biogerontology 5:1-10

33. Cristofalo VJ (2005) SA- $\beta$-Gal staining: biomarker or delusion. Exp Gerontol 40:836-838

34. Moiseeva O, Mallette FA, Mukhopadhyay UK, Moores A, Ferbeyre G (2006) DNA damage signaling and p53-dependent senescence after prolonged beta-Interferon stimulation. Mol Biol Cell 17:1583-1592

35. Freshney R (2000) Culture of Animal Cells, 4th edn. Wiley, New York 